\title{
Systems of Inequalities Characterizing Ring Homomorphisms
}

\author{
Włodzimierz Fechner ${ }^{1}$ and Andrzej Olbryśs ${ }^{2}$ \\ ${ }^{1}$ Institute of Mathematics, Łódź University of Technology, Ul. Wólczańska 215, 93-005 Łódź, Poland \\ ${ }^{2}$ Institute of Mathematics, University of Silesia, Bankowa 14, 40-007 Katowice, Poland
}

Correspondence should be addressed to Włodzimierz Fechner; wlodzimierz.fechner@p.lodz.pl

Received 16 August 2016; Accepted 1 November 2016

Academic Editor: Shanhe Wu

Copyright (C) 2016 W. Fechner and A. Olbryś. This is an open access article distributed under the Creative Commons Attribution License, which permits unrestricted use, distribution, and reproduction in any medium, provided the original work is properly cited.

Assume that $T: \mathscr{P} \rightarrow \mathscr{R}$ and $U: \mathscr{P} \rightarrow \mathscr{R}$ are arbitrary mappings between two partially ordered rings $\mathscr{P}$ and $\mathscr{R}$. We study a few systems of functional inequalities which characterize ring homomorphisms. For example, we prove that if $T$ and $U$ satisfy $T(f+g) \geq T(f)+T(g), U(f \cdot g) \geq U(f) \cdot U(g)$, for all $f, g \in \mathscr{P}$ and $T \geq U$, then $U=T$ and this mapping is a ring homomorphism. Moreover, we find two other systems for which we obtain analogous assertions.

\section{Introduction}

Let $X$ be a compact Hausdorff topological space. By $C(X)$ we denote the space of all continuous real valued functions defined on $X$ and equipped with the supremum norm. Rădulescu [1] showed that if an operator $T: C(X) \rightarrow C(X)$ is super-additive and super-multiplicative simultaneously, that is, it satisfies the system

$$
\begin{gathered}
T(f+g) \geq T(f)+T(g), \\
T(f \cdot g) \geq T(f) \cdot T(g),
\end{gathered}
$$

for each $f, g \in C(X)$, then there exist a clopen subset $B \subseteq X$ and a continuous function $\varphi: X \rightarrow X$ such that

$$
T(f)=\chi_{B} \cdot f \circ \varphi,
$$

where $\chi$ denotes the characteristic function of a given set. In particular, $T$ is linear, multiplicative, and continuous. Ercan [2] has shown that Rădulescu's assumption that $X$ is a compact Hausdorff space may be dropped. More results on system (1) and on related questions have been obtained by Dhombres [3], Volkmann [4, 5], J. X. Chen and Z. L. Chen [6], Gusić [7], and the first author [8,9], among others.

Our purpose is to generalize system (1) to the case of two unknown operators $T$ and $U$ in various directions. Moreover, in our last result we provide a condition sufficient for the separation of two mappings by a linear and multiplicative operator.

\section{Main Results}

To the end of the section let $(\mathscr{P}, \leq)$ and $(\mathscr{R}, \leq)$ be partially ordered rings and let $T: \mathscr{P} \rightarrow \mathscr{R}$ and $U: \mathscr{P} \rightarrow \mathscr{R}$ be two arbitrary mappings. We will need three crucial assumptions (cf. [3]):

(A1) Every nonnegative element of $\mathscr{P}$ is a square:

$$
\left[f=g^{2}\right], \quad \forall f \in \mathscr{P}, f \geq 0, \exists g \in \mathscr{P} .
$$

(A2) Every square in $\mathscr{R}$ is nonnegative:

$$
\left[g^{2} \geq 0\right], \quad \forall g \in \mathscr{R} \text {. }
$$

(A3) 0 is the only element in $\mathscr{R}$ whose square is equal to 0 :

$$
\left[\left(g^{2}=0\right) \Longrightarrow(g=0)\right], \quad \forall g \in \mathscr{R}
$$

We will begin with the following system:

$$
\begin{gathered}
T(f+g) \geq T(f)+T(g), \\
U(f \cdot g) \geq U(f) \cdot U(g),
\end{gathered}
$$

assumed for all $f, g \in \mathscr{P}$. Note that (6) with $T=U$ becomes (1). 
In our first theorem we assume that $U \leq T$ (i.e., $U(f) \leq$ $T(f)$ in ring $\mathscr{R}$ for every $f \in \mathscr{P})$. We will prove that under assumptions $(A 1),(A 2)$, and $(A 3)$ system (6) together with inequality $U \leq T$ characterizes ring homomorphisms; that is, $T=U$ and $T$ is simultaneously additive and multiplicative.

Theorem 1. Assume that conditions ( $A 1),(A 2)$, and $(A 3)$ hold true. Then $T$ and $U$ satisfy (6) and $U \leq T$ if and only if $T=U$ and $T$ is a ring homomorphism.

Proof. We will prove the nontrivial implication only. First, put $f=g=0$ into both inequalities and apply assumption $(A 2)$ to get $T(0) \leq 0$ and $U(0) \geq U(0)^{2} \geq 0$. Since $U \leq T$, then $U(0)=T(0)=0$. obtain

Next, substitute $f=g$ into the second inequality to

$$
U\left(g^{2}\right) \geq U(g)^{2} \geq 0, \quad g \in \mathscr{P} .
$$

Using $(A 1)$ we derive

$$
f \geq 0 \Longrightarrow U(f) \geq 0 .
$$

Now, let $g=-f$ in the first inequality. We have

$$
0=T(0)=T(f-f) \geq T(f)+T(-f), \quad f \in \mathscr{P} .
$$

Consequently, using this we deduce

$$
U(-f) \leq T(-f) \leq-T(f) \leq-U(f), \quad f \in \mathscr{P} .
$$

Next, put $g=-f$ in the second inequality of (6). We get

$$
U\left(-f^{2}\right) \geq U(f) \cdot U(-f), \quad f \in \mathscr{P} .
$$

On the other hand, (10) implies that

$$
U\left(f^{2}\right)+U\left(-f^{2}\right) \leq 0, \quad f \in \mathscr{P} .
$$

Join the last two inequalities and use (7) to derive

$$
0 \geq U\left(f^{2}\right)+U\left(-f^{2}\right) \geq U(f)[U(f)+U(-f)],
$$

$$
f \in \mathscr{P} \text {. }
$$

Replace in (13) $f$ by $-f$ to arrive at

$$
0 \geq U(-f)[U(f)+U(-f)], \quad f \in \mathscr{P} .
$$

Then add (13) and (14) side-by-side to reach

$$
[U(f)+U(-f)]^{2} \leq 0, \quad f \in \mathscr{P} .
$$

Due to the assumptions $(A 2)$ and $(A 3)$ the last inequality is equivalent to the equality $U(f)+U(-f)=0$ for every $f \in$ $\mathscr{P}$; that is, $U$ is odd. Having this, it is easy to see that (10) implies that $T=U$. To get the additivity of $T$ it is enough to apply the first inequality of the system with $f$ replaced by $-f$ and $g$ replaced by $-g$ and use the oddness of $T$. Similarly, substitution $f \rightarrow-f$ in the second inequality leads to the conclusion that $U$ is multiplicative.
Remark 2. The assumption $U \leq T$ in Theorem 1 is essential and cannot be replaced by the opposite inequality. What is more, it is clear that every even super-additive mapping $T$ is nonpositive, whereas every even super-multiplicative mapping $U$ is nonnegative; therefore $T \leq 0 \leq U$. In particular, both mappings are trivially separated by the zero ring homomorphism (c.f. Theorem 12).

In our next result we will deal with the system:

$$
\begin{gathered}
T(f+g) \geq U(f)+T(g), \\
U(f \cdot g) \geq T(f) \cdot T(g),
\end{gathered}
$$

postulated for all $f, g \in \mathscr{P}$. In this case we do not need to assume an inequality between $T$ and $U$.

Theorem 3. Assume that conditions (A1), (A2), and (A3) hold true and $\mathscr{P}$ has a unit 1 . Then $T$ and $U$ satisfy (16) if and only if $T=U$ and $T$ is a ring homomorphism.

Proof. Again, one implication is obvious. To prove the nontrivial one put $g=0$ in the first inequality of (16) to get

$$
U(f) \leq T(f)-T(0), \quad f \in \mathscr{P} .
$$

Next, let $f=g$ in the second inequality of (16) and use (A2) to arrive at

$$
0 \leq[T(f)]^{2} \leq U\left(f^{2}\right), \quad f \in \mathscr{P} .
$$

Therefore, thanks to $(A 1)$ we have $U(f) \geq 0$ whenever $f \geq$ 0 . In particular, $U(0) \geq 0$. On the other hand, from (17) we obtain

$$
U(0) \leq T(0)-T(0)=0 .
$$

Consequently, $U(0)=0$. Having this and using (18) we see that

$$
0 \leq T(0)^{2} \leq U(0)=0
$$

so $T(0)=0$. Thus, $(17)$ reduces to

$$
U(f) \leq T(f), \quad f \in \mathscr{P} .
$$

Next, apply the second inequality of (16) with $g$ replaced by $-g$. Then, add the result to this inequality side-by-side and then use (21) and the first inequality:

$$
\begin{aligned}
& T(f)[T(g)+T(-g)] \leq U(f \cdot g)+U(-f \cdot g) \\
& \quad \leq T(f \cdot g)+U(-f \cdot g) \leq T(f \cdot g-f \cdot g) \\
& \quad=T(0)=0, \quad f, g \in \mathscr{P} .
\end{aligned}
$$

Now, apply the above estimate for $f$ replaced by $-f$ and add to the original one side-by-side:

$$
[T(f)+T(-f)][T(g)+T(-g)] \leq 0, \quad f, g \in \mathscr{P} .
$$

In particular, $[T(f)+T(-f)]^{2} \leq 0$, which in view of $(A 2)$ and (A3) leads to $T(f)=-T(f)$ for all $f \in \mathscr{P}$; that is, $T$ is odd. 
From (21) and from the second inequality of (16) we deduce that

$$
T(f) T(g) \leq U(f \cdot g) \leq T(f \cdot g), \quad f, g \in \mathscr{P} .
$$

Replace $g$ by $-g$ and apply the oddness of $T$ to derive the reverse inequality:

$$
\begin{aligned}
-T(f) T(g) & =T(f) T(-g) \leq T(-f \cdot g) \\
& =-T(f \cdot g), \quad f, g \in \mathscr{P} .
\end{aligned}
$$

Therefore, $T$ is multiplicative. Using this, from the second inequality of (16) we get

$$
T(f \cdot g)=T(f) T(g) \leq U(f \cdot g), \quad f, g \in \mathscr{P} .
$$

Since $1 \in \mathscr{P}$, then we obtain from this $T \leq U$, which is the opposite inequality to (21). Therefore $T=U$ and to finish the proof it is enough to apply Theorem 1.

In what follows we will study one more system:

$$
\begin{aligned}
& T(f+g) \geq U(f)+U(g), \\
& U(f \cdot g) \geq T(f) \cdot T(g),
\end{aligned}
$$

postulated for all $f, g \in \mathscr{P}$. It turns out that in general (27) is not equivalent to (16), but some arguments which worked for (16) can be utilized for (27). Again, no inequality between $T$ and $U$ will be assumed.

Lemma 4. Assume that conditions (A1) and (A2) hold true. If $T$ and $U$ satisfy (27), then

(i) $U(f) \geq 0$ for $f \geq 0$,

(ii) $U(f) \leq T(f)$ for $f \in \mathscr{P}$.

Proof. Since the second inequalities of (16) and (27) are identical, then from the proof of Theorem 3 we derive (i). In particular, $U(0) \geq 0$. Next, put $g=0$ in the first inequality of (27) to get

$$
U(f) \leq T(f)-U(0) \leq T(f), \quad f \in \mathscr{P} .
$$

Theorem 5. Assume that conditions ( $A 1),(A 2)$, and $(A 3)$ hold true. Then $T$ and $U$ satisfy (27) and $T(0)=0$ if and only if $T=U$ and $T$ is a ring homomorphism.

Proof. We will justify the nontrivial implication. By repeating the respective calculations of the proof of Theorem 3 which involved the second inequality only together with $U \leq T$ and $T(0)=0$, we get that $T$ is odd and then, following this proof further, that $T$ is multiplicative and also $T=U$.

An easy example shows that, even in the case the target space is the real line, the assumption $T(0)=0$ cannot be dropped.
Example 6. For arbitrary $c \in[0,1 / 2]$ functions $T: \mathscr{P} \rightarrow \mathbb{R}$ and $U: \mathscr{P} \rightarrow \mathbb{R}$ given by $T(f)=c$ and $U(f)=(1 / 2) c$ for $f \in \mathscr{P}$ satisfy (27). Therefore, assumption $T(0)=0$ in Theorem 5 is essential. In fact, both functions are subadditive.

Further, if $U$ is constant and equal to some $c<1 / 4$, then for every function $T: \mathscr{P} \rightarrow \mathbb{R}$ such that $2 c \leq T(f) \leq \sqrt{c}$ for all $f \in \mathscr{P}$ the pair $(T, U)$ solves (27). Similarly, if $T$ is constant and equal to some $d<1 / 2$, then for every mapping $U: \mathscr{P} \rightarrow \mathbb{R}$ such that $d^{2} \leq U(f) \leq(1 / 2) d$ for all $f \in$ $\mathscr{P}$ the pair $(T, U)$ solves (27). Nonconstant solutions can be provided in a similar fashion by giving some small "freedom" for both functions.

In what follows, we will state some observations for real solutions of (21), which are not covered by the previous theorem, that is, such that $T(0) \neq 0$.

Proposition 7. Assume that condition $(A 1)$ holds true, $\mathscr{R}=\mathbb{R}$ and the pair $(T, U)$ solves $(27)$. If $T(0) \neq 0$, then $T(f) \leq 1 / 2$ and $U(f) \leq 1 / 4$ for every $f \in \mathscr{P}$.

Proof. Put $g=f$ in both inequalities of (27) to get $2 U(f) \leq$ $T(2 f)$ and $T(f)^{2} \leq U\left(f^{2}\right)$ for all $f \in \mathscr{P}$. Therefore, we obtain

$$
2 T(f)^{2} \leq T\left(2 f^{2}\right), \quad f \in \mathscr{P} .
$$

Clearly $T(0)>0$. Now, suppose that $T(f)>1 / 2$ for some $f$. We claim that $\sup T(\mathscr{P})=+\infty$. Let $\varepsilon:=T(f)-1 / 2>0$. From (29) we deduce the estimate

$$
\begin{aligned}
T\left(2 f^{2}\right) & \geq 2 T(f)^{2}=2\left(\frac{1}{2}+\varepsilon\right)^{2} \\
& =\left(\frac{1}{2}+\varepsilon\right)+\varepsilon(1+2 \varepsilon)>T(f)+\varepsilon
\end{aligned}
$$

which justifies our claim. Therefore, there exists a sequence $g_{n} \in \mathscr{P}$ such that $T\left(g_{n}\right)>n$ for $n \in \mathbb{N}$. From this and from the second inequality of (27) we derive that

$$
0<T(0) \leq \frac{U(0)}{T\left(g_{n}\right)} \longrightarrow 0
$$

as $n \rightarrow \infty$, which leads to a contradiction. $1 / 2$.

If $U(f)>1 / 4$ for some $f \in \mathscr{P}$, then $T(2 f) \geq 2 U(f)>$

In view of Proposition 7, the next one is self-evident.

Proposition 8. Assume that condition $(A 1)$ holds true, $\mathscr{R}=$ $\mathbb{R}$, the pair $(T, U)$ solves $(27)$, and $T(0) \neq 0$. Denote $A:=$ $U^{-1}(1 / 4)$ and $B:=T^{-1}(1 / 2)$. Then

$$
\begin{aligned}
& f, g \in A \Longrightarrow f+g \in B, \\
& f, g \in B \Longrightarrow f \cdot g \in A .
\end{aligned}
$$

In particular,

$$
\begin{aligned}
& f, g \in A \Longrightarrow(f+g)^{2} \in A, \\
& f, g \in B \Longrightarrow 2(f \cdot g) \in B .
\end{aligned}
$$


Next, we will show that if $\mathscr{P}$ contains a unit, then $T$ and $U$ attain positive values only.

Proposition 9. Assume that condition (A1) holds true, $\mathscr{P}$ contains a unit $1, \mathscr{R}=\mathbb{R}$, and the pair $(T, U)$ solves (27). If $T(0) \neq 0$, then $T(f)>0$ and $U(f)>0$ for every $f \in \mathscr{P}$.

Proof. Put $g=1$ into the second inequality of (27) to get

$$
T(f) \cdot T(1) \leq U(f) \leq T(f), \quad f \in \mathscr{P} .
$$

Therefore, since $T(1) \leq 1 / 2$, then $T(f) \geq 0$ and since $1=$ $1^{2} \geq 0$, then also $U(f) \geq 0$ for all $f \in \mathscr{P}$.

Now, suppose that $U\left(f_{0}\right)=0$ for some $f_{0} \in \mathscr{P}$. Then from (34) we have

$$
T\left(f_{0}\right) T(1) \leq U\left(f_{0}\right)=0
$$

so $T\left(f_{0}\right)=0$. Consequently,

$$
0 \leq U(0)+U\left(f_{0}\right) \leq T\left(f_{0}\right)=0
$$

and thus $U(0)=0$. Finally, (34) implies that

$$
T(0) \cdot T(1) \leq U(0)=0 ;
$$

therefore $T(0)=0$, which is a contradiction.

Example 6 shows that it can happen that both sets $A, B$ defined in Proposition 8 are empty, even if mappings $T, U$ are regular. In what follows we will show that under some additional assumptions if one of the sets is nonempty, then both are equal to $\mathscr{P}$; that is, $T$ and $U$ are constant and equal to $1 / 2$ and $1 / 4$, respectively.

Corollary 10. Assume that condition (A1) holds true, $\mathscr{P}$ contains a unit $1, \mathscr{R}=\mathbb{R}$, the pair $(T, U)$ solves (27), and the sets $A, B$ are defined as in Proposition 8. If $T(0) \neq 0$ and $B$ contains an invertible element, then $A=B=\mathscr{P}$.

Proof. Let $\beta \in B$ be an invertible element. Apply the second inequality of (27) for $g=\beta$ to get $(1 / 2) T(f) \leq U(\beta f)$ for all $f \in \mathscr{P}$. Join this with the first inequality to obtain

$$
\begin{aligned}
T(f)+T(g) & \leq 2 U(\beta f)+2 U(\beta g) \\
& \leq 2 T(\beta(f+g)), \quad f, g \in \mathscr{P} .
\end{aligned}
$$

Now, suppose that $T$ is nonconstant. Define $a=\inf T(\mathscr{P})$. Clearly, $0 \leq a<1 / 2$. Take $h \in \mathscr{P}$ such that $T(h)<(1 / 2) a+$ $1 / 4$. Put $f:=\beta^{-1} h-\beta$ and apply (38) with $g=\beta$ to get

$$
a+\frac{1}{2} \leq T\left(\beta^{-1} h-\beta\right)+\frac{1}{2} \leq 2 T(h)<a+\frac{1}{2},
$$

a contradiction. Thus $T=1 / 2$ on $\mathscr{P}$. Equivalently, $B=\mathscr{P}$ and, as a consequence, we get

$$
A=B \cdot B \subseteq 1 \cdot B=\mathscr{P} .
$$

Corollary 11. Assume that condition $(A 1)$ holds true, $\mathscr{P}$ contains a unit $1, \mathscr{R}=\mathbb{R}$, the pair $(T, U)$ solves (27), and the sets $A$, $B$ are defined as in Proposition 8. If $T(0)=1 / 2$ and $A$ contains an invertible element, then $A=B=\mathscr{P}$.

Proof. We have $0 \in B$, so by Proposition 8 also $0 \in A$. Consequently $A \subseteq B$ and we can apply Corollary 10 .

Now, let $X$ be a nonempty set and let $\mathscr{B}(X)$ denote the space of all bounded real valued functions defined on $X$ and equipped with the supremum norm. We consider $\mathscr{B}(X)$ with an order relation defined, as usual, coordinatewise; that is,

$$
f \leq g \Longleftrightarrow f(x) \leq g(x), \quad x \in X, f, g \in \mathscr{B}(X) .
$$

We will terminate the paper with an application of Theorem 1 to a separation problem. We will give a sufficient condition for the separation of two operators $T, U: \mathscr{B}(X) \rightarrow$ $\mathscr{B}(X)$ by operator $\Phi: \mathscr{B}(X) \rightarrow \mathscr{B}(X)$ which is additive and multiplicative simultaneously. Note that if $\mathscr{P}=\mathscr{R}=\mathscr{B}(X)$, then the conditions $(A 1)-(A 3)$ are satisfied.

Theorem 12. Let $U, T: \mathscr{B}(X) \rightarrow \mathscr{B}(X)$ satisfy the inequality

$$
\sum_{j=1}^{m} \prod_{k=1}^{n_{j}} U\left(f_{k}^{j}\right) \leq T\left(\sum_{j=1}^{m} \prod_{k=1}^{n_{j}} f_{k}^{j}\right),
$$

for all $m \in \mathbb{N}, f_{1}^{1}, \ldots, f_{n_{1}}^{1}, f_{1}^{2}, \ldots, f_{n_{2}}^{2}, \ldots, f_{1}^{m}, \ldots, f_{n_{m}}^{m} \in$ $\mathscr{B}(X)$. Then there exists an additive and multiplicative operator $\Phi: \mathscr{B}(X) \rightarrow \mathscr{B}(X)$ such that

$$
U(f) \leq \Phi(f) \leq T(f), \quad f \in \mathscr{B}(X) .
$$

Proof. Let us define the operator $\Phi: \mathscr{B}(X) \rightarrow \mathbb{R}^{X}$ by the formula

$$
\begin{gathered}
\Phi(f):=\sup \left\{\sum_{j=1}^{m} \prod_{k=1}^{n_{j}} U\left(f_{k}^{j}\right) \mid f=\sum_{j=1}^{m} \prod_{k=1}^{n_{j}} f_{k}^{j}, m\right. \\
\left.\in \mathbb{N}, f_{1}^{1}, \ldots, f_{n_{1}}^{1}, \ldots, f_{1}^{m}, \ldots, f_{n_{m}}^{m} \in \mathscr{B}(X)\right\} .
\end{gathered}
$$

Directly from the definition and from condition (42) we infer that

$$
U(f) \leq \Phi(f) \leq T(f), \quad f \in \mathscr{B}(X),
$$

and consequently, $\Phi$ attains values in $\mathscr{B}(X)$. We shall prove that operator $\Phi$ is super-additive and super-multiplicative. Take arbitrary $f, g \in \mathscr{B}(X)$ and arbitrary $\alpha, \beta \in \mathscr{B}(X)$ such that

$$
\begin{aligned}
& \alpha<\Phi(f), \\
& \beta<\Phi(g) .
\end{aligned}
$$

By the definition of operator $\Phi$, there exist $m \in \mathbb{N}$, $f_{1}^{1}, \ldots, f_{n_{1}}^{1}, \ldots, f_{1}^{m}, \ldots, f_{n_{m}}^{m} \in \mathscr{B}(X)$ such that

$$
f=\sum_{j=1}^{m} \prod_{k=1}^{n_{j}} f_{k}^{j}
$$


and there exist $p \in \mathbb{N}, g_{1}^{1}, \ldots, g_{r_{1}}^{1}, \ldots, g_{1}^{p}, \ldots, g_{r_{p}}^{p} \in \mathscr{B}(X)$ such that

$$
\begin{aligned}
& g=\sum_{i=1}^{p} \prod_{s=1}^{r_{i}} g_{s}^{i}, \\
& \alpha<\sum_{j=1}^{m} \prod_{k=1}^{n_{j}} U\left(f_{k}^{j}\right), \\
& \beta<\sum_{i=1}^{p} \prod_{s=1}^{r_{i}} U\left(g_{s}^{i}\right) .
\end{aligned}
$$

Obviously,

$$
\begin{array}{r}
f+g=\sum_{t=1}^{m+p} \prod_{l=1}^{c_{t}} h_{l}^{t}, \\
f g=\sum_{u=1}^{m p} \prod_{v=1}^{m_{u}} \gamma_{v}^{u},
\end{array}
$$

where

$$
h_{l}^{t}, \gamma_{v}^{u} \in \bigcup_{j=1}^{m} \bigcup_{k=1}^{n_{j}}\left\{f_{k}^{j}\right\} \cup \bigcup_{i=1}^{p} \bigcup_{s=1}^{r_{i}}\left\{g_{s}^{i}\right\}
$$

for all $t=1, \ldots, m+p, l=1, \ldots, c_{t}$ and $u=1, \ldots, n p, v=$ $1, \ldots, m_{u}$.

Therefore, we get

$$
\begin{aligned}
\alpha+\beta & <\sum_{j=1}^{m} \prod_{k=1}^{n_{j}} U\left(f_{k}^{j}\right)+\sum_{i=1}^{p} \prod_{s=1}^{n_{i}} U\left(g_{s}^{j}\right)=\sum_{t=1}^{m+p} \prod_{l=1}^{n_{t}} U\left(h_{l}^{t}\right) \\
& \leq \Phi(f+g) .
\end{aligned}
$$

On the other hand,

$$
\begin{gathered}
\min \{\alpha \beta, \alpha \Phi(g), \Phi(f) \Phi(g), \beta \Phi(f)\} \\
<\left(\sum_{j=1}^{m} \prod_{k=1}^{n_{j}} U\left(f_{k}^{j}\right)\right)\left(\sum_{i=1}^{p} \prod_{s=1}^{n_{i}} U\left(g_{s}^{i}\right)\right) \\
=\sum_{u=1}^{m p} \prod_{v=1}^{m_{u}} U\left(\gamma_{v}^{u}\right) \leq \Phi(f \cdot g) .
\end{gathered}
$$

We can choose $\alpha$ and $\beta$ such that for every $x \in X$ the values $\alpha(x)$ and $\beta(x)$ are arbitrarily close to $\Phi(f)(x)$ and $\Phi(g)(x)$, respectively. Therefore, we get

$$
\begin{gathered}
\Phi(f)+\Phi(g) \leq \Phi(f+g), \\
\Phi(f) \cdot \Phi(g) \leq \Phi(f \cdot g),
\end{gathered}
$$

for all $f, g \in \mathscr{B}(X)$. To finish the proof it remains to apply Theorem 1 .

\section{Competing Interests}

The authors declare that there is no conflict of interests regarding the publication of this paper.

\section{References}

[1] M. Rădulescu, "On a supra-additive and supra-multiplicative operator of $C(X)$," Bulletin Mathématique de la Société des Sciences Mathématiques de Roumanie, Nouvelle Serie, vol. 24, no. 72, pp. 303-305, 1980

[2] Z. Ercan, "A remark on supra-additive and supra-multiplicative operators on C(X)," Mathematica Bohemica, vol. 132, pp. 55-58, 2007.

[3] J. Dhombres, "Sur les fonctions simultanement suradditives et surmultiplicatives," Comptes Rendus Mathématiques, vol. 5, no. 5, pp. 207-210, 1983.

[4] P. Volkmann, "Sur un système d'inéquations fonctionnelles," Comptes Rendus Mathématiques, vol. 4, pp. 155-158, 1982.

[5] P. Volkmann, "Sur les fonctions simultanément suradditives et surmultiplicatives," Bulletin Mathématique de la Société des Sciences Mathématiques de Roumanie. Nouvelle Série, vol. 28, no. 76, pp. 181-184, 1984.

[6] J. X. Chen and Z. L. Chen, "On supra-additive and supramultiplicative maps," Journal of Function Spaces and Applications, vol. 2013, Article ID 108535, 3 pages, 2013.

[7] I. Gusić, "A note on certain maps between ordered fields," Journal of Mathematical Inequalities, vol. 3, no. 4, pp. 657-661, 2009.

[8] W. Fechner, "Inequalities characterizing linear-multiplicative functionals," Journal of Function Spaces, vol. 2015, Article ID 945758, 3 pages, 2015.

[9] W. Fechner, "Inequalities connected with averaging operators. Part II," Indagationes Mathematicae, vol. 26, no. 1, pp. 40-49, 2015. 


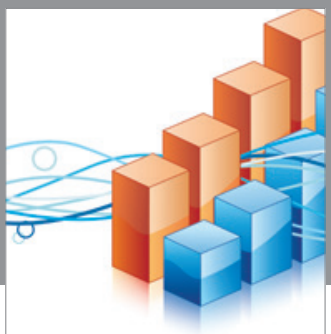

Advances in

Operations Research

vatem alat4

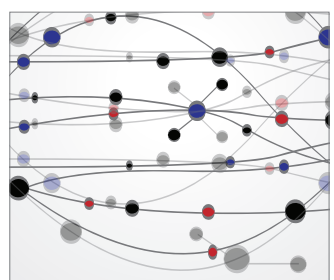

\section{The Scientific} World Journal
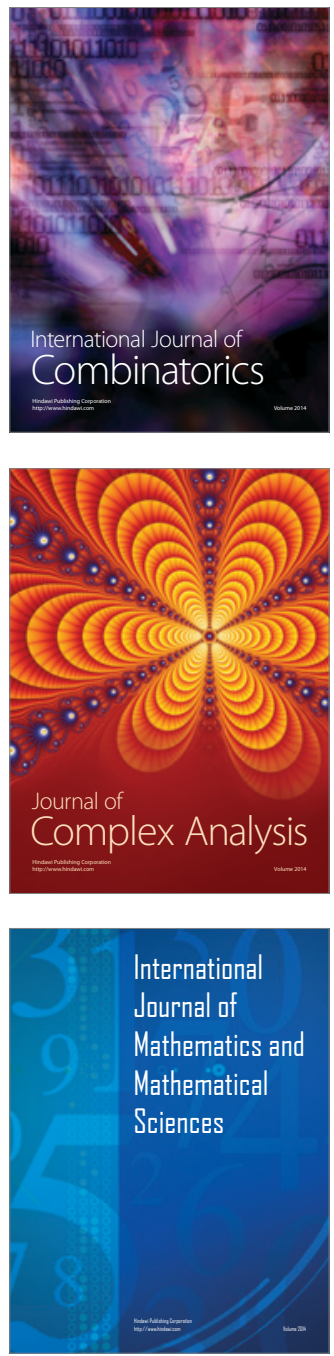
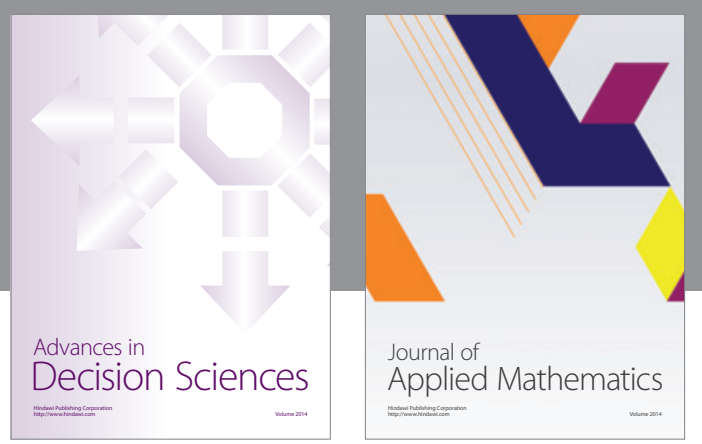

Algebra

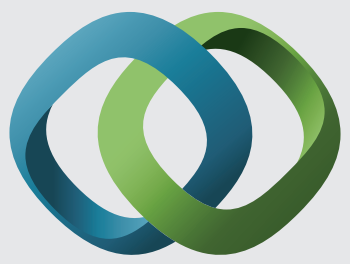

\section{Hindawi}

Submit your manuscripts at

http://www.hindawi.com
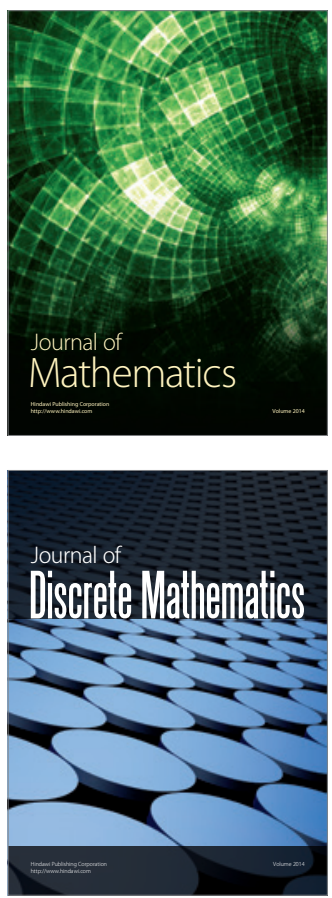

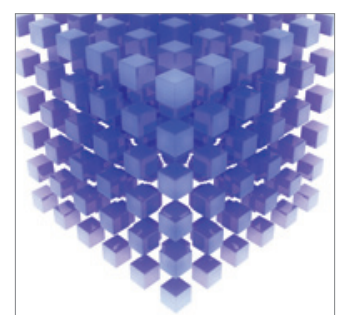

Mathematical Problems in Engineering
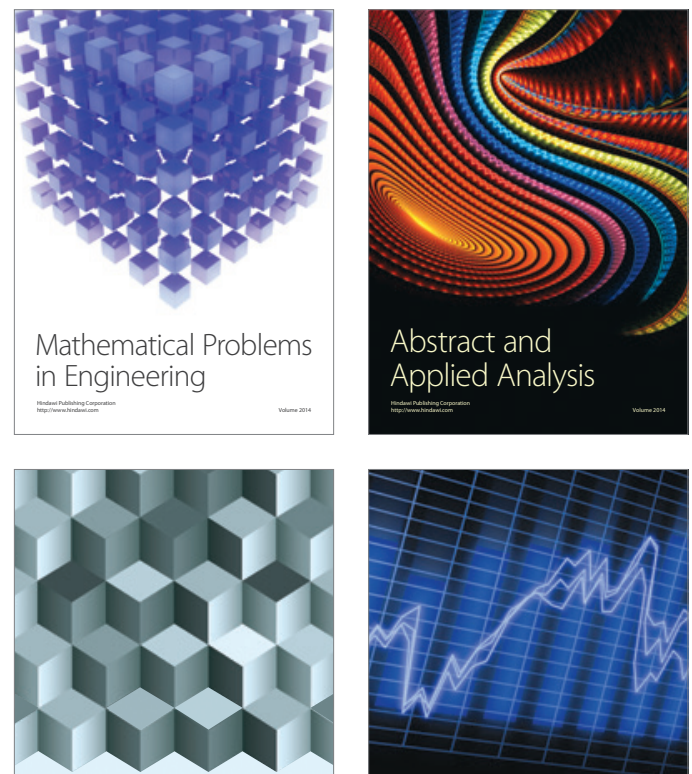

Journal of

Function Spaces

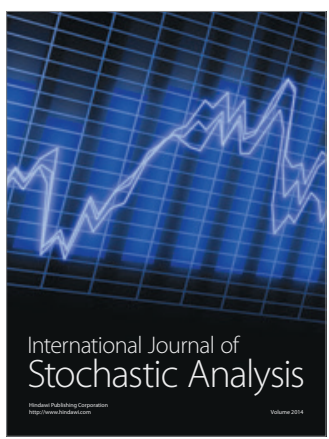

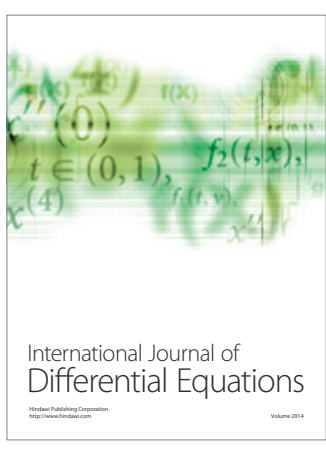
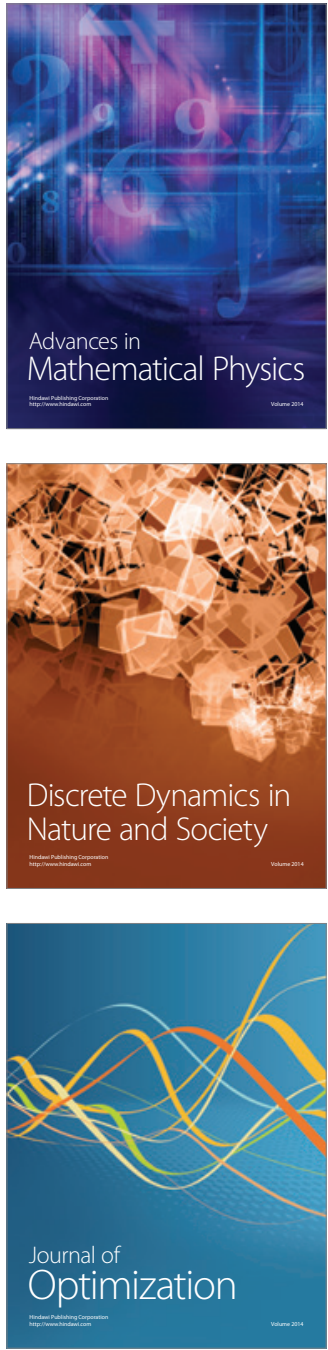